\title{
Guaranteed Cost Bounds for Robust Stability and Performance Analysis of Discrete-Time Systems
}

Scot L. Osburn and Dennis S. Bernstein ${ }^{1}$

Department of Aerospace Engineering, The University of Michigan

\begin{abstract}
In this paper we derive new guaranteed cost bounds for robust stability and performance with real structured uncertainty for discrete-time systems. In particular, we obtain a shifted bounded real bound, a linear bound, a shifted linear bound, an inverse bound, a shifted inverse bound, and a shifted Popov bound. Several examples are used to compare these new bounds.
\end{abstract}

\section{Introduction}

The analysis and synthesis of robust controller has been of intense interest during the past three decades [1]. While unstructured complex-valued uncertainty can be addressed nonconservatively using quadratic bounds [2], structured real-valued uncertainty is a more difficult problem due to the discontinuity of the structurcd singular value and stability margins [3]. The most effective approach to this problem has been the development of frequencydependent scales and multipliers that account for phase restrictions on the parametric or dynamic uncertainty $[4,5]$.

For stability analysis with real polytopic uncertainty, LMI techniques can be used to solve multiple Lyapunov equations to determine stability bounds [6]. This approach avoids the need for frequency-dependent multipliers and utilizes convex optimization methods to obtain common Lyapunov functions.

An alternative approach that is applicable to controller synthesis is Riccati-based methods which provide guaranteed cost bounds for the worst-case $\mathrm{H}_{2}$ performance. For continuous-time systems a large class of guaranteed cost bounds have been developed. While the small gain bounds are the best known due to their connections with the small gain theorem $[7,8]$, alternative bounds have been developed as well [9|-(13). For structured real-valued uncertainty, these bounds have significantly reduced conservatism as compared to small gain bounds.

For discrete-time systems quadratic bounds have been developed in the context of bounded-real theory (see [14] and the references given therein). Discrete-time Popov bounds given in $[15,16]$ also provide quadratic tests for robust stability and performance. For a sampled-data system with parametric uncertainty, a nonquadratic bound was developed in [17]. Compared to continuous-time sys tems, however, there has been relatively little effort devoted to the development of discrete-time bounds.

The objective of the present paper is to develop novel bounds for structured real uncertainty for discrete-time systems. Some of these bounds can be viewed as the counterpart of bounds developed for continuous-time systems in [13]. Unlike [17] we consider general uncertainty structures rather than sampled-data uncertainty structures. We consider parameter-independent bounds. each equivalent to a common Lyapunov function, and parameterdependent bounds which are equivalent to multiple Lyapunov functions.

In Section 2 we present the main robustness result that provides the basis for specific bounds given later in the paper. In Section 3 we present an LMI approach to bounding polytopic uncertainties. In Sections $4,5,6$, and 7 , we present the discrete-time forms of the shifted bounded real bound. the linear and shifted linear bounds. the inverse and shifted inverse bounds. and the shifted

\footnotetext{
IThis research was supported in part by the Air Force Office of Scientific Research This reacarch was supportod
under grant F49620-98-1-0037.
}

Popov Bound. Finally, in Section 8, we present several numerical examples to compare the different bounds.

$\begin{array}{ll}\text { Notation } \\ \mathbf{R}^{d} & d \times 1 \text { real column vectors } \\ \mathbf{R}^{m \times n} & m \times n \text { real matrices } \\ I_{n}, \mathbf{N}^{n} & n \times n \text { identity matrix, nonnegative-definite matrices } \\ \mathbf{S}^{n}, \mathbf{P}^{n} & n \times n \text { symmetric matrices, positive-definite matrices } \\ A \leq B & B-A \text { is nonnegative definite } \\ \mathcal{E}(\cdot) & \text { expectation operator } \\ \mathrm{tr} & \text { trace operator }\end{array}$

$|H| \quad\left(H H^{\mathrm{T}}\right)^{\frac{1}{2}}$, where $H \in \mathbf{R}^{n \times n}$

2. Robust Performance and Guaranteed Cost Bounds Let $U \subset \mathbf{R}^{n \times n}$ denote an uncertainty set and consider the discretetime system

$$
x(k+1)=(A+\Delta A) x(k)+D w(k),
$$

where $x \in \mathbf{R}^{n}$ and $w \in \mathbf{R}^{d}$ are the state and disturbance, respectively, $\Delta A \in \mathcal{U}$, and the disturbance $w$ is a standard zero-mean white noise process. We assume throughout that $A$ is asymptotically stable. Next, consider the performance variable

$$
z(k)=E x(k) .
$$

If $A+\Delta A$ is asymptotically stable for all $\Delta A \in \mathcal{U}$, then define the worst-case $\mathrm{H}_{2}$ performance measure

$$
J(\mathcal{U})=\sup _{\Delta A \in \mathcal{U}} \operatorname{tr} P_{\Delta A} V
$$

where $V \triangleq D D^{\mathrm{T}}$ and $P_{\triangle A}$ is the nonnegative-definite solution to the Lyapunov equation

$$
P_{\Delta A}=(A+\Delta A)^{\mathrm{T}} P_{\Delta A}(A+\Delta A)+R,
$$

where $R \triangleq E^{\mathrm{T}} E$.

The following result, which is an extension of Theorem 3.1 of [18], provides a bound for the worst-case cost $J(\mathcal{U})$.

Theorem 1 Let $\Omega: \mathcal{N} \subseteq \mathbf{S}^{n} \rightarrow \mathbf{S}^{n}$ be such that there exists $P \in \mathcal{N}$ satisfying

$$
P=A^{\mathrm{T}} P A+\Omega(P)+R,
$$

and let $P_{0}: \mathcal{U} \rightarrow \mathrm{S}^{n}$ be such that

$$
0 \leq P+P_{0}(\Delta A), \Delta A \in \mathcal{U} \text {. }
$$

and

$$
\begin{aligned}
0 \leq & \Omega(P)-\left[(A+\Delta A)^{\mathrm{T}} P_{0}(\Delta A)(A+\Delta A)-P_{0}(\Delta A)\right] \\
& -\left[\Delta A^{\mathrm{T}} P A+A^{\mathrm{T}} P \Delta A+\Delta A^{\mathrm{T}} P \Delta A\right], \Delta A \in \mathcal{U}
\end{aligned}
$$

Then $(A+\triangle A, E)$ is detectable for all $\Delta A \in \mathcal{U}$ if and only if $A+\Delta A$ is asymptotically stable for all $\Delta A \in \mathcal{U}$. In this case.

$$
P_{\Delta A} \leq P+P_{0}(\Delta A), \Delta A \in \mathcal{U},
$$

where $P_{\Delta A} \in \mathbb{N}^{n}$ is given by (4), and

$$
J(\mathcal{U}) \leq \operatorname{tr} P V+\sup _{\Delta A \in \mathcal{U}} \operatorname{tr} P_{0}(\Delta A) V .
$$

If, in addition, there exists $\bar{P}_{0} \in \mathrm{S}^{n}$ such that

$$
P_{0}(\Delta A) \leq \bar{P}_{0}, \quad \Delta A \in \mathcal{U},
$$

then

$$
J(\mathcal{U}) \leq \operatorname{tr}\left[\left(P+\bar{P}_{0}\right) V\right]
$$


The pair $\left(\Omega, P_{0}\right)$ is a bound. A bound is parameter-independent if $P_{0}$ is constant. In this case, we write $P_{0}$ for $P_{0}(\Delta A)$ and set $\bar{P}_{0}=P_{0}$. Finally, a bound is parameter-dependent if $P_{0}$ depends on $\Delta A$. For a given bound $\left(\Omega, P_{0}\right)$, the following result yields an equivalent bound $\left(\hat{\Omega}, \hat{P}_{0}\right)$.

Proposition 1 Let $\Omega: \mathcal{N} \subseteq \mathrm{S}^{n} \rightarrow \mathrm{S}^{n}, P \in \mathcal{N}$, and $P_{0}: \mathcal{U} \rightarrow \mathbf{S}^{n}$ satisfy (5)-(7), and let $\bar{P}_{0} \in \mathbf{S}^{n}$ satisfy (10). Let $\overline{\hat{P}}_{0} \in \mathbf{S}^{n}$, and define $\hat{\mathcal{N}} \subseteq \mathrm{S}^{n}, \hat{\Omega}: \hat{\mathcal{N}} \rightarrow \mathrm{S}^{n}$ and $\hat{P}_{0}: \mathcal{U} \rightarrow \mathrm{S}^{n}$ by $\hat{\mathcal{N}} \triangleq \mathcal{N}+\bar{P}_{0}-\overline{\hat{P}}_{0}$,

$$
\hat{\Omega}\left(P+\bar{P}_{0}-\overline{\hat{P}}_{0}\right) \triangleq \Omega(P)-A^{\mathrm{T}}\left(\bar{P}_{0}-\overline{\hat{P}}_{0}\right) A+\left(\bar{P}_{0}-\overline{\hat{P}}_{0}\right),
$$

and

$$
\hat{P}_{0}(\Delta A) \triangleq P_{0}(\Delta A)-\vec{P}_{0}+\overrightarrow{\hat{P}}_{0} .
$$

Then Theorem 1 is satisfied with $\mathcal{N}, \Omega, P, P_{0}$, and $\vec{P}_{0}$ replaced by $\hat{\mathcal{N}}, \hat{\Omega}, P+\bar{P}_{0}-\overrightarrow{\hat{P}}_{0}, \dot{P}_{0}$, and $\hat{\hat{P}}_{\mathrm{0}}$.

Remark 1 In Proposition 1, if $P_{0}(\Delta A)=P_{0}=\bar{P}_{0}, \Delta A \in \mathcal{U}$, then $\hat{P}_{0}(\Delta A)=\hat{P}_{0}=\hat{\hat{P}}_{0}, \Delta A \in \mathcal{U}$. In particular, choosing $\overline{\hat{P}}_{0}=0$ implies $\hat{P}_{0}(\Delta A)=0, \Delta A \in \mathcal{U}$.

\section{LMI's for Robust Performance}

In this section we use linear matrix inequalities to determine the least conservative parameter-independent bound for robust stability and performance with polytopic uncertainty. Let $U$ have the form

$$
\mathcal{U}=\left\{\Delta A: \Delta A=\sum_{i=1}^{r} \delta_{i} A_{i}, \text { where }\left|\delta_{i}\right| \leq \gamma, i=1, \ldots, r\right\},
$$

where $A_{1}, \ldots, A_{r} \in \mathbf{R}^{n \times n}$.

Proposition 2 Let $P \in N^{n}$ satisfy the $2^{r}$ constraints

$\left(A+\gamma A_{1}+\cdots+\gamma A_{r}\right)^{\mathrm{T}} P\left(A+\gamma A_{1}+\cdots+\gamma A_{r}\right)-P+R \leq 0$, $\left(A-\gamma A_{1}+\cdots+\gamma A_{r}\right)^{\mathrm{T}} P\left(A-\gamma A_{1}+\cdots+\gamma A_{r}\right)-P+R \leq 0$,

$\left(A-\gamma A_{1}-\cdots+\gamma A_{r}\right)^{\mathrm{T}} P\left(A-\gamma A_{1}-\cdots+\gamma A_{r}\right)-P+R \leq 0$, $\left(A-\gamma A_{1}-\cdots-\gamma A_{r}\right)^{\mathrm{T}} P\left(A-\gamma A_{1}-\cdots-\gamma A_{\mathrm{r}}\right)-P+R \leq 0$.

Then

$$
(A+\Delta A)^{\mathrm{T}} P(A+\Delta A)-P+R \leq 0, \Delta A \in \mathcal{U} .
$$

Then $(A+\Delta A, E)$ is detectable for all $\triangle A \in U$ if and only if $A+\Delta A$ is asymptotically stable for all $\Delta A \in \mathcal{U}$. In this case,

$$
P_{\Delta A} \leq P, \Delta A \in \mathcal{U} \text {. }
$$

Remark 2 Minimizing the convex objective $J(\mathcal{U})=\operatorname{tr} P V$ subject to the LMI's given in Proposition 2, is a convex optimization problem. In addition, the optimal cost $J(\mathcal{U})$ from this optimization provides the lowest possible cost for a parameter-independent bound under polytopic uncertainty

\section{Shifted Bounded Real Bound}

Let the uncertainty set $U$ be given by

$$
\mathcal{U}=\left\{\Delta A: \Delta A=B_{0} F C_{0}, \text { where } F \in \mathcal{F}\right\},
$$

where $\mathcal{F}$ is a subset of

$$
\mathcal{F}_{\mathrm{BR}} \triangleq\left\{F \in \mathbf{R}^{k_{1} \times k_{2}}: F^{\mathrm{T}} F \leq M\right\},
$$

where $M \in \mathbf{N}^{k_{2}}, B_{0} \in \mathbf{R}^{n \times k_{1}}$. and $C_{0} \in \mathbf{R}^{k_{2} \times n}$. The following result concerns the classical bounded real bound $[19,20,15]$.
Proposition 3 Define $\mathcal{N}=\left\{P \in \mathrm{N}^{n}: B_{0}^{\mathrm{T}} P B_{0}<I\right\}$. Suppose there exists $P \in \mathcal{N}$ satisfying

$$
P=A^{\mathrm{T}} P A+A^{\mathrm{T}} P B_{0}\left(I-B_{0}^{\mathrm{T}} P B_{0}\right)^{-1} B_{0}^{\mathrm{T}} P A+C_{0}^{\mathrm{T}} M C_{0}+R .
$$

Then (6) and (7) are satisfied with $P_{0}=0$.

Next we present a variation of Proposition 3, the shifted bounded real bound.

Proposition 4 Let $T \in \mathrm{P}^{k_{1}}, N, H \in \mathbf{R}^{k_{1} \times n}$, and $Z \in \mathrm{S}^{n}$. Define $\mathcal{N}=\left\{P \in \mathbb{N}^{n}: B_{0}^{\mathrm{T}} P B_{0}<T\right\}$. Suppose there exists $P \in \mathcal{N}$ satisfying

$P=A_{\mathrm{s}}^{\mathrm{T}} P A_{\mathrm{s}}+\left(A_{\mathrm{s}}^{\mathrm{T}} P B_{0}-H^{\mathrm{T}}\right)\left(T-B_{0}^{\mathrm{T}} P B_{0}\right)^{-1}\left(B_{0}^{\mathrm{T}} P A_{\mathrm{s}}-H\right)+Z+R$,

where

$$
\begin{aligned}
\left(N^{\mathrm{T}} T N+\right. & \left.H^{\mathrm{T}} N+N^{\mathrm{T}} H\right)+\left(N^{\mathrm{T}} T+H^{\mathrm{T}}\right) F C_{0} \\
& +C_{0}^{\mathrm{T}} F^{\mathrm{T}}(T N+H)+C_{0}^{\mathrm{T}} F^{\mathrm{T}} T F C_{0} \leq \mathcal{Z}, F \in \mathcal{F}
\end{aligned}
$$

and $A_{\mathrm{s}} \triangleq A-B_{0} N$. Then (6) and (7) are satisfied with $P_{0}=0$.

Note that the shifted bounded real equation (18) with $H=0$, $T=I, N=0$ and $Z=C_{0}^{\mathbf{T}} M C_{0}$ yields the bounded real bound equation (17). Letting $H=0$ and $T=I$, we obtain the the discrete-time version of the continuous-time shifted bounded real bound [21]

Corollary 1 Define $\mathcal{N}=\left\{P \in \mathbb{N}^{n}: B_{0}^{\mathbf{T}} P B_{0}<I\right\}$. Suppose there exists $P \in \mathcal{N}$ satisfying

$$
P=A_{\mathrm{s}}^{\mathrm{T}} P A_{\mathrm{s}}+A_{\mathrm{s}}^{\mathrm{T}} P B_{0}\left(I-B_{0}^{\mathrm{T}} P B_{0}\right)^{-1} B_{0}^{\mathrm{T}} P A_{\mathrm{s}}+M_{\mathrm{s}}+R,
$$

where $M_{s} \in \mathbf{N}^{n}$ satisfies

$$
N^{\mathbf{T}} N+N^{\mathbf{T}} F C_{0}+C_{0}^{\mathbf{T}} F^{\mathbf{T}} N+C_{0}^{\mathbf{T}} F^{\mathbf{T}} F C_{0} \leq M_{\mathrm{B}}, F \in \mathcal{F} .
$$

Then (6) and (7) are satisfied with $P_{0}=0$.

Next, assume that $\mathcal{U}$ is given by (14), where $A_{1}, \ldots, A_{\tau} \in \mathrm{R}^{n \times n}$. Furthermore, let $B_{i} \in \mathbf{R}^{\mathbf{n} \times k_{i}}$ and $C_{i} \in \mathbf{R}^{k_{i} \times n}$ satisfy $A_{i}=B_{i} C_{i}$, $i=1, \ldots, r$, and let

$$
B_{0}=\left[\begin{array}{lll}
B_{1} & \cdots & B_{r}
\end{array}\right] \in \mathbf{R}^{n \times k}, \quad C_{0}=\left[\begin{array}{c}
C_{1} \\
\vdots \\
C_{r}
\end{array}\right] \in \mathbf{R}^{k \times n},
$$

where $k=\sum_{i=1}^{r} k_{i}$. Then $\Delta A=B_{0} F C_{0}$, where $F \in \mathcal{F}$, and $\mathcal{F}$ is given by

$\mathcal{F}=\left\{F=\right.$ block $-\operatorname{diag}\left(\delta_{1} I_{k_{1}}, \ldots, \delta_{r} I_{k_{r}}\right) \in \mathbf{R}^{k \times k}$ where $\left.\delta_{i} \in[-\gamma, \gamma]\right\}$,

and $M$ in (16) can be chosen to be $\gamma^{2} I_{k}$. Note that $\mathcal{F}$ is a proper subset of $\mathcal{F}_{\mathrm{BR}}$.

One choice for $M_{\mathrm{g}}$ is given by

$$
M_{\mathrm{s}}=N^{\mathrm{T}} N+\gamma\left|N^{\mathrm{T}} C_{0}+C_{0}^{\mathrm{T}} N\right|+\gamma^{2} C_{0}^{\mathrm{T}} C_{0},
$$

where $|H|=\left(H H^{\mathrm{T}}\right)^{1 / 2}$. With this choice of $M_{\mathrm{s}},(20)$ becomes

$$
\begin{aligned}
P= & A_{\mathrm{s}}^{\mathrm{T}} P A_{\mathrm{s}}+A_{\mathrm{s}}^{\mathrm{T}} P B_{0}\left(I-B_{0}^{\mathrm{T}} P B_{0}\right)^{-1} B_{0}^{\mathrm{T}} P A_{\mathrm{s}} \\
& +N^{\mathrm{T}} N+\gamma\left|N^{\mathrm{T}} C_{0}+C_{0}^{\mathrm{T}} N\right|+\gamma^{2} C_{0}^{\mathrm{T}} C_{0}+R .
\end{aligned}
$$

Similarly, (19) is satisfied with

$$
\begin{aligned}
Z= & \left(N^{\mathrm{T}} T N+H^{\mathrm{T}} N+N^{\mathrm{T}} H\right) \\
& +\gamma\left|\left(N^{\mathrm{T}} T+H^{\mathrm{T}}\right) C_{0}+C_{0}^{\mathrm{T}}(T N+H)\right|+\gamma^{2} C_{0}^{\mathrm{T}} T C_{0} .
\end{aligned}
$$


Proposition 5 Suppose $r=1, A=A^{\mathrm{T}}$ is invertible, and $A_{1}=$ $-A_{1}^{\mathrm{T}}$. Furthermore, suppose there exists $m>0$, such that

$$
\frac{1}{m} A R A<A-A\left(\left[A+\gamma^{2} A_{1}^{\mathrm{T}} A^{-1} A_{1}\right]\right) A .
$$

Then

$$
P=m\left(A+\gamma^{2} A_{1}^{\mathrm{T}} A^{-1} A_{1}\right)+R
$$

is a solution to (18) where $B_{0}=A, C_{0}=A^{-1} A_{1} N=0, T=m A$, and $H=T$. Furthermore, if $P \geq 0$ then $(6)$ and $(7)$ are satisfied with $P_{0}=0$.

\section{Shifted Linear Bound}

In this section, we let the uncertainty set $U$ be given by (14). The following result provides the discrete-time form of the continuoustime linear bound [22]-[24].

Proposition 6 Define $A_{0}=\sum_{i=1}^{r} A_{i}$. Let $0<\alpha<1$, and suppose there exists $P \in \mathrm{N}^{n}$ satisfying

$$
P=\frac{1}{1-\alpha} A^{\mathrm{T}} P A+\frac{\gamma^{2}}{\alpha} A_{0}^{\mathrm{T}} P A_{0}+R .
$$

Then (6) and (7) are satisfied with $P_{0}=0$.

The solution to (26) can be written as

$$
P=\operatorname{vec}^{-1}\left(\left[I-\frac{1}{1-\alpha}\left(A^{\mathrm{T}} \otimes A^{\mathrm{T}}\right)-\frac{\gamma^{\mathrm{T}}}{\alpha}\left(A_{0}^{\mathrm{T}} \otimes A_{0}^{\mathrm{T}}\right\rangle\right]^{-1} \operatorname{vec} R\right) .
$$

Next we obtain the shifted linear bound, the discrete-time version of the continuous-time shifted linear bound [13].

Proposition 7 Define $A_{0}=\sum_{i=1}^{r} A_{i}$. Let $\alpha>0, N \in \mathrm{S}^{n}$, and define $\mathcal{N}=\left\{P \in \mathbf{N}^{n}:(1-\alpha)(P-N) \geq 0\right\}$. Suppose there exists $P \in \mathcal{N}$ satisfying

$$
\begin{aligned}
P= & \frac{1}{1-\alpha} A^{\mathrm{T}} P A+\frac{\gamma^{2}}{\alpha} A_{0}^{\mathrm{T}} P A_{0}-\frac{a}{1-\alpha} A^{\mathrm{T}} N A-\frac{1-a}{\alpha} \gamma^{2} A_{0}^{\mathrm{T}} N A_{0} \\
& +\gamma \sum_{i=1}^{\mathrm{T}}\left|A_{i}^{\mathrm{T}} N A+A^{\mathrm{T}} N A_{i}\right|+R
\end{aligned}
$$

Then (6) and (7) are satisfied with $P_{0}=0$.

\section{Shifted Inverse Bound}

In this section we let the uncertainty set $\mathcal{U}$ be given by (14). The following result provides a discrete-time form of the inverse bound [10].

Proposition 8 Let $\alpha>0$, suppose $A$ is invertible, and suppose there exists $P \in \mathbf{P}^{n}$ satisfying

$$
\begin{aligned}
P= & (1+\gamma \alpha r) A^{\mathrm{T}} P A+R+\gamma \sum_{i=1}^{r}\left[\gamma A_{i}^{\mathrm{T}} P A_{i}\right. \\
& \left.+\frac{1}{4 \alpha}\left(A_{i}^{\mathrm{T}} P A+A^{\mathrm{T}} P A_{i}\right)\left(A^{\mathrm{T}} P A\right)^{-1}\left(A_{i}^{\mathrm{T}} P A+A^{\mathrm{T}} P A_{i}\right)\right]
\end{aligned}
$$

Then (6) and (7) are satisfied with $P_{0}=0$.

Note that (28) can be written as

$$
\begin{aligned}
P= & (1+\gamma \alpha r) A^{\mathrm{T}} P A+\sum_{i=1}^{\Gamma} A^{\mathrm{T}}\left[\frac{\gamma}{4 \alpha}\left(\tilde{A}_{i}^{\mathrm{T}} P+P \tilde{A}_{i}\right)\right. \\
& \left.\times P^{-1}\left(\tilde{A}_{i}^{\mathrm{T}} P+P \tilde{A}_{i}\right)+\gamma^{2} \tilde{A}_{i}^{\mathrm{T}} P \tilde{A}_{i}\right] A+R,
\end{aligned}
$$

where $\tilde{A}_{i}=A_{i} A^{-1}$. Next we obtain the shifted inverse bound. the discrete-time version of the continuous-time shifted inverse bound [13].
Proposition 9 Let $\alpha>0, M, N \in \mathrm{S}^{n}$, suppose $A$ is invertible, and suppose there exists $P \in \mathbf{N}^{n} \cap\left(\mathbf{P}^{n}+N\right)$ satisfying

$$
\begin{aligned}
P= & (1+\alpha \gamma) A^{\mathrm{T}} P A+\sum_{i=1}^{r} A^{\mathrm{T}}\left(\gamma^{2} \tilde{A}_{i}^{\mathrm{T}} P \tilde{A}_{i}-\alpha \gamma N\right. \\
& +\frac{\gamma}{\alpha}\left[\tilde{A}_{i}^{\mathrm{T}}(P-M)+(P-M) \tilde{A}_{i}\right](P-N)^{-1} \\
& \left.\times\left[\tilde{A}_{i}^{\mathrm{T}}(P-M)+(P-M) \tilde{A}_{i}\right]+\gamma\left|\tilde{A}_{i}^{\mathrm{T}} M+M \tilde{A}_{i}\right|\right) A+R
\end{aligned}
$$

Then (6) and (7) are satisfied with $P_{0}=0$.

If $N=M$ then (29) becomes

$$
\begin{aligned}
P= & (1+\alpha \gamma) A^{\mathrm{T}} P A+\sum_{i=1}^{r} A^{\mathrm{T}}\left(\gamma^{2} \tilde{A}_{i}^{\mathrm{T}} P \tilde{A}_{i}-\alpha \gamma N\right. \\
& +\frac{\gamma}{\alpha}\left[\tilde{A}_{i}^{\mathrm{T}}(P-N)+(P-N) \tilde{A}_{i}\right](P-N)^{-1} \\
& \left.\times\left[\bar{A}_{i}^{\mathrm{T}}(P-N)+(P-N) \bar{A}_{i}\right]+\gamma\left|\tilde{A}_{i}^{\mathrm{T}} N+N \bar{A}_{i}\right|\right) A+R
\end{aligned}
$$

With $N=M=0$, the shifted bound (28) is obtained. Alternatively, with $M=0$, (29) becomes

$$
\begin{aligned}
P= & (1+\alpha \gamma) A^{\mathrm{T}} P A+\sum_{i=1}^{r} A^{\mathrm{T}}\left(\gamma^{2} \tilde{A}_{i}^{\mathrm{T}} P \tilde{A}_{i}-\alpha \gamma N\right. \\
& \left.+\frac{\gamma}{\alpha \alpha}\left[\tilde{A}_{i}^{\mathrm{T}} P+P \tilde{A}_{i}\right](P-N)^{-1}\left[\tilde{A}_{i}^{\mathrm{T}} P+P \tilde{A}_{i}\right]\right) A+R
\end{aligned}
$$

\section{Shifted Popov Bound}

Define the Popov uncertainty set $\mathcal{F}_{\text {Pop }}$ as

$$
\mathcal{F}_{\mathrm{Pop}} \triangleq\left\{F \in \mathrm{S}^{k}: M_{\mathrm{L}} \leq F \leq M_{\mathrm{U}}\right\}
$$

where $M_{\mathrm{L}}, M_{\mathrm{U}} \in \mathrm{S}^{k}$ are such that $M \triangleq M_{\mathrm{U}}-M_{\mathrm{L}}$ is positive definite. The following result concerns the Popov bound $[15,16]$. Let $\mathcal{U}$ be given by (15), where $\mathcal{F} \subseteq \mathcal{F}_{\text {Pop }}$.

Proposition 10 Let $N \in \mathbf{R}^{k \times k}$ and $\mu \in \mathbf{S}^{k}$ satisfy

$$
N^{\mathbf{T}}\left(F-M_{L}\right)=\left(F-M_{L}\right) N \leq \mu, \quad F \in \mathcal{F},
$$

$R_{0} \triangleq 2 M^{-1}-N C_{0} B_{0}-\left(N C_{0} B_{0}\right)^{\mathbf{T}}-B_{0}^{\mathbf{T}} C_{0}^{\mathbf{T}} \mu C_{0} B_{0}-B_{0}^{\mathbf{T}} P B_{0}>0$.

Furthermore, suppose there exists $P \in \mathbf{P}^{\mathbf{n}}$ satisfying

$$
\begin{aligned}
P= & \tilde{A}_{\text {Pop }}^{\mathrm{T}} P \tilde{A}_{\text {Pop }}+\left(\tilde{A}_{\text {Pop }}-I\right)^{\mathrm{T}} C_{0}^{\mathrm{T}} \mu C_{0}\left(\tilde{A}_{\text {Pop }}-I\right)+R \\
& +\left[C_{0}+\left(N C_{0}+B_{0}^{\mathrm{T}} C_{0}^{\mathrm{T}} \mu C_{0}\right)\left(\tilde{A}_{\text {Pop }}-I\right)+B_{0}^{\mathrm{T}} P \tilde{A}_{\text {Pop }}\right]^{\mathrm{T}} \\
& \times R_{0}^{-1}\left[C_{0}+\left(N C_{0}+B_{0}^{\mathrm{T}} C_{0}^{\mathrm{T}} \mu C_{0}\right)\left(\tilde{A}_{\mathrm{Pop}}-I\right)+B_{0}^{\mathrm{T}} P \tilde{A}_{\mathrm{Pop}}\right]
\end{aligned}
$$

where $\bar{A}_{\text {Pop }} \triangleq A+B_{0} M_{\mathrm{L}} C_{0}$. Then (7) is satisfied with $P_{0}: \mathcal{U} \rightarrow \mathrm{S}^{n}$ given by

$$
P_{0}(\Delta A)=C_{0}^{\mathrm{T}}\left(F-M_{L}\right) N C_{0} .
$$

Remark $3 \vec{P}_{0}=C_{0}^{\mathbf{T}} \mu C_{0}$ satisfies (10).

Next we present a shifted Popov bound. Let $\mathcal{U}$ be given by (15) where $\mathcal{F} \subseteq \mathcal{F}_{\text {Pop }}$.

Proposition 11 Let $N \in \mathbf{R}^{k \times k}, \mu \in \mathbf{S}^{k}, Z \in \mathbf{N}^{n}$ and $H \in \mathbf{P}^{k}$ satisfy

$$
\begin{gathered}
\left(F-M_{L}\right) N=N^{\mathrm{T}}\left(F-M_{L}\right) \leq \mu, \quad F \in \mathcal{F}, \\
H\left(F-M_{\mathrm{L}}\right) N=N^{\mathrm{T}}\left(F-M_{\mathrm{L}}\right) H, \quad F \in \mathcal{F}, \\
H\left(F-M_{\mathrm{L}}\right)=\left(F-M_{\mathrm{L}}\right) H, \quad F \in \mathcal{F},
\end{gathered}
$$

$$
\begin{aligned}
R_{0} \triangleq & 2 M^{-1}-N C_{0} B_{0} H^{-1}-\left(N C_{0} B_{0} H^{-1}\right)^{\mathrm{T}} \\
& -H^{-1} B_{0}^{\mathrm{T}} C_{0}^{\mathrm{T}} H \mu C_{0} B_{0} H^{-1}-H^{-1} B_{0}^{\mathrm{T}} P B_{0} H^{-1}>0
\end{aligned}
$$


and

$$
\begin{aligned}
{\left[B_{0} X^{\mathrm{T}}(F-\right.} & \left.\left.M_{L}\right) H C_{0}+C_{0}^{\mathrm{T}} H\left(F-M_{L}\right) X B_{0}^{\mathrm{T}}\right] \\
& +\left(A_{\text {Pop }}-I\right)^{\mathbf{T}} C_{0}^{\mathrm{T}} H \mu C_{0}\left(A_{\mathrm{Pop}}-I\right) \leq Z, \quad F \in \mathcal{F} .
\end{aligned}
$$

Furthermore, suppose there exists $P \in \mathrm{P}^{n}$ satisfying

$$
\begin{array}{rl}
P=A_{\text {Pop }}^{\mathrm{T}} & P A_{\text {Pop }}+\left[H C_{0}+\left(N C_{0}+H^{-1} B_{0}^{\mathrm{T}} C_{0}^{\mathrm{T}} H \mu C_{0}\right)\left(A_{\text {Pop }}-I\right)\right. \\
& \left.+H^{-1} B_{0}^{\mathrm{T}} P A_{\mathrm{P}_{\mathrm{op}}}-X B_{0}^{\mathrm{T}}\right]^{\mathrm{T}} R_{0}^{-1}\left[H C_{0}+H^{-1} B_{0}^{\mathrm{T}} P A_{\mathrm{Pop}}\right. \\
& \left.+\left(N C_{0}+H^{-1} B_{0}^{\mathrm{T}} C_{0}^{\mathrm{T}} H \mu C_{0}\right)\left(A_{\mathrm{Pop}}-I\right)-X B_{0}^{\mathrm{T}}\right] \\
+ & +Z
\end{array}
$$

Then (7) is satisfied with $P_{0}: \mathcal{U} \rightarrow \mathrm{S}^{n}$ given by

$$
P_{0}(\Delta A)=C_{0}^{\mathrm{T}} H\left(F-M_{L}\right) N C_{0} .
$$

Remark 4 Proposition 11, gives the discrete-time version of the continuous-time shifted Popov bound [13]

Remark 5 Setting $X=0, Z=0$, and $H=I$ in Proposition 11 yields Proposition 10 .

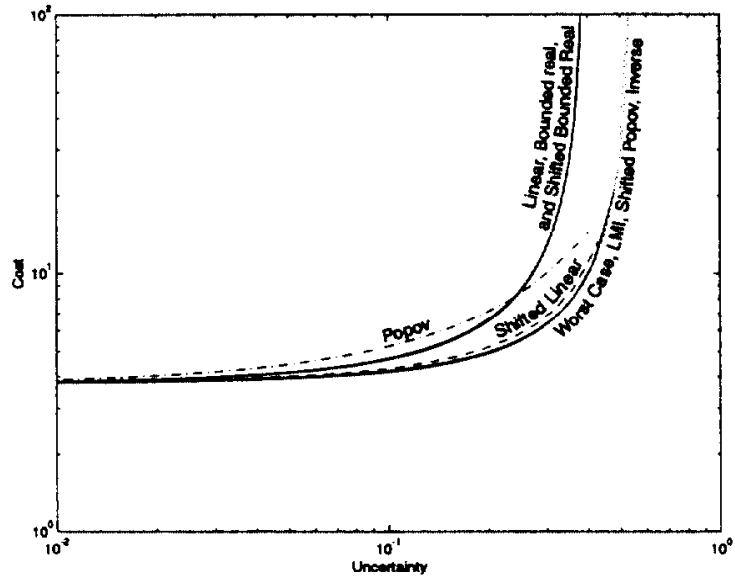

Figure 1: Cost bounds for Example 1.

\section{Examples}

LMI methods can be used to calculate solutions along with optimal scalings for bounds such as the linear, bounded real, inverse, and Popov bounds, as well as their shifted counterparts. For more on the setup of LMI's for solving bounds, see [13]. In the numerical examples that follow, LMI methods were used to obtain the best parameter-independent bound along with the Popov and shifted Popov bounds.

\section{Example 1 Let}

$$
A=\left[\begin{array}{cc}
0.523 & -0.307 \\
0.307 & 0.523
\end{array}\right], \quad A_{0}=\left[\begin{array}{cc}
0 & 1 \\
-1 & 0
\end{array}\right] \text {. }
$$

Furthermore, let $R=\left[\begin{array}{cc}0.25 & 0.12 \\ 0.12 & .3\end{array}\right]$, and $V=\left[\begin{array}{ll}4 & 1 \\ 1 & 4\end{array}\right]$. Figure 1 shows the actual worst case cost, along with the best possible parameter-independent bound given by Remark 2. Next, let $B_{0}$ and $C_{0}$ be given by

$$
B_{0}=\left[\begin{array}{cc}
0 & \varepsilon^{-1} \\
\varepsilon^{-1} & 0
\end{array}\right], \quad C_{0}=\left[\begin{array}{cc}
-\varepsilon & 0 \\
0 & \varepsilon
\end{array}\right]
$$

where $\varepsilon \neq 0$ is a free parameter used for optimization. The bounded real bound, shifted bounded real, linear, and Popov bound, predict stability for $|\delta| \leq 0.4$. Figure 1 shows the inverse bound and the shifted linear bound, with $N=\beta I$, predicting stability for $|\delta|<0.45$, with the inverse bound giving a tight fit to the worst case cost in this range. Finally, the shifted Popov bound predicts stability for $|\delta|<0.48$, with performance coinciding with the actual worst case performance in that region.

Example 2 Consider the problem given in Example 1, with $A=$ $\left[\begin{array}{cc}0.523 & -0.307 \\ -0.307 & 0.523\end{array}\right]$. Figure 2 shows the actual worst case cost, along with the best possible parameter-independent bound given by Remark 2 . In this example, the inverse and Popov bounds are conservative predicting stability for $|\delta| \leq 0.035$ and $|\delta|<0.11$, respectively. The bounded real bound and linear bound predict stability for $|\delta|<0.17$ and $|\delta|<0.27$ respectively. The shifted bounded real bound given in Proposition 5 performs slightly better than the linear bound giving better performance for $|\delta|<0.28$. The shifted linear bound with $N=\beta I$ extends the predicted stability range to $|\delta|<0.4$, which falls short of the LMI bound prediction of $|\delta|<0.45$. In contrast, the shifted Popov bound predicts stability beyond the LMI bound, up to $|\delta|<0.48$.

Example 3 Finally, consider the problem given in Example 2 with $A_{0}=\left[\begin{array}{ll}0 & 1 \\ 0 & 0\end{array}\right]$. Figure 3 shows the actual worst case cost, along with the best possible parameter-independent bound given by Remark 2 . The inverse bound predicts stability for $|\delta| \leq 0.07$ and shifted inverse bound with $M=0$ and $N=\beta \operatorname{diag}(0,1)$ predicts stability for $|\delta|<0.125$. The Popov bound predicts stability for $|\delta|<0.125$ with increased performance. Finally, the shifted Popov bound predicts stability for $|\delta|<0.425$ and coincides with the performance prediction of the LMI bound.

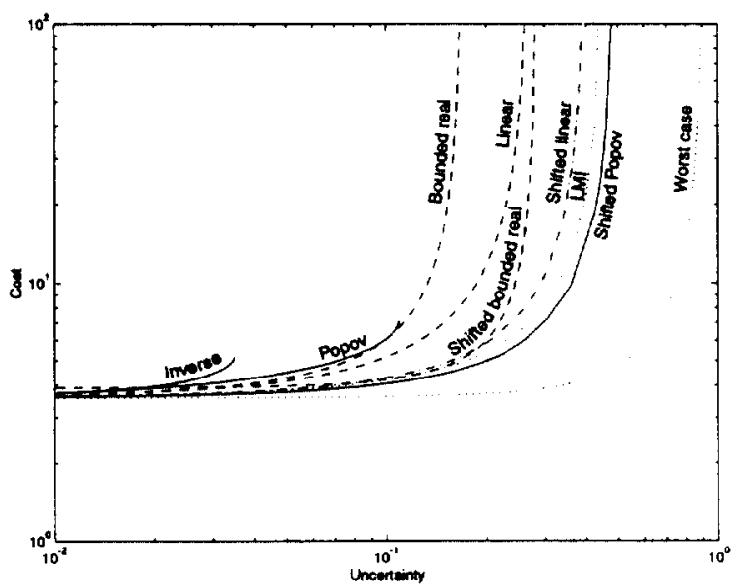

Figure 2: Cost bounds for Example 2.

\section{References}

[1] K. Zhou, Robust and Optimal Control, Prentice Hall, 1996.

[2] A. Packard and J. C. Doyle, "The complex structured singular value," Automatica, Vol 29, pp. 71-109, 1993.

[3] B. Barmish, P. Khargonekar. Z. Shi, and R. 'Tempo, "Robustness Margins Need Not be a Continuous Function of the Problem Data," Systems and Control Letters, Vol. 15, pp. 91-98, 1990

[4] M. K. H. Fan, A. L. Tits and J. C. Duyle, "Robustmess in the Presence of Mixed Parametric Uncertainty and Unmodeled Dynamics," IEEE TAC, V. 36, 25-38, 1991. 
[5] A. L. Tits, V. Balakrishnan, and L. Lee, "Robustness Under Bounded Uncertainty with Phase Information," IEEE TAC, V 44, 50-65 1999.

[6] S. Boyd, L. E. Ghaoui, E. Feron, V. Balakrishnan, Linear Matrix Inequalities in System and Control Theory, SIAM, Philadclphia, 1994.

[7] I. R. Petersen and C. V. Hollot, "A Riccati Equation Approach to the Stabilization of Uncertain Systems," Automatica, Vol. 22, pp. 397-411, 1986.

[8] P. P. Khargonekar, I. R. Petersen and K. Zhou, "Robust Stability of Uncertain Linear Systems: Quadratic Stability and $\mathrm{H}_{\infty}$ Control Theory," IEEE Trans. Autom. Contr., Vol. 35, pp. 356-361, 1990.

[9] D. S. Bernstein and W. M. Haddad, "Robust Stability and Performance via Fixed-Order Dynamic Compensation with Guaranteed Cost Bounds," Math. Contr. Sig. Sys., Vol. 3, pp. 139-163, 1990.

[10] D. S. Bernstein and W. M. Haddad, "Robust Stability and Performance Analysis for State Space Systems via Quadratic Lyapunov Bounds," SIAM J. Matrix Anal. Appl., Vol. 11, pp. 239-271, 1990.

[11] S. L. Osburn and D. S. Bernstein, "A Review and Comparison of Guaranteed Cost Bounds for Robust Stability and Performance," Proc. IEEE Conf. Dec. Cantr., pp. 787-788, San Diego, CA, December 1997.

[12] S. L. Osburn and D. S. Bernstein, "Robust Stability and Performance Analysis with Skew-Symmetric Uncertainties Using the Shifted Bounded Real Bound," Proc. Conf. Dec. Contr., pp. 4392-4393, Tampa, FL. December 1998.

[13] S. L. Osburn and D. S. Bernstein, "Robust Stability and Performance Analysis Using Shifted Quadratic and Nonquadratic Guaranteed Cost Bounds," Proc. Amer. Contr. Conf., San Diego, CA, June 1999.

[14] C. K. Chui and G. Chen, Discrete $H_{\infty}$ Optimization, Springer-Verlag, Berlin, Second edition, 1997.

(15] W. M. Haddad and D. S. Bernstein, "Explicit Construction of Quadratic Lyapunov Functions for the Small Gain, Positivity, Circle, and Popov Theorems and Their Application to Robust Stability, Part II: Discrete-Time Theory," Int. J. Robust and Nonlinear Control, Vol. 4, pp. 249-265, 1994.

[16] W. M. Haddad and D. S. Bernstein, "Parameter-Dependent Lyapunov Functions and the Discrete-Time Popov Criterion for Robust Analysis," Automatica, Vol. 30. pp. 1015-1021, 1994.

[17] D. S. Bernstein and C. V. Hollot, "Robust Stability for Sampled-Data Control Systems," Sys. Contr. Lett., Vol. 13, pp. 217-226. 1989.

[18] W. M. Haddad and D. S. Bernstein, "Parameter-Dependent Lyapunov Functions and the Popov Criterion in Robust Analysis and Synthesis." IEEE Trans. Autom. Contr., Vol. 40, pp. $536-543,1995$

[19] P. P. Vaidyanathan, "The Discrete-Time Bounded-Real Lemma in Digital Filtering," IEEE Trans. Circ. Sys., Vol. 32, pp. 918-924, 1985.
[20] P. Agathoklis, E. I. Jury, and M. Mansour, "The DiscreteTime Strictly Bounded-Real Lemma and the Computation of Positive Definite Solutions to the 2-D Lyapunov Equations," IEEE Trans. Circ. Sys., Vol. 36, pp. 830-837, 1989.

[21] F. Tyan and D. S. Bernstein, "Shifted Quadratic Guaranteed Cost Bounds for Robust Stability and Performance," Proc. 13th IFAC World Congress, Vol. G: Education, Robust Control I, pp. 285-290, San Francisco, CA, July 1996.

[22] B. N. Jain, "Guaranteed error estimation in Uncertain Systems," IEEE Trans. Autom. Contr., pp. 230-232, 1975.

[23] D. S. Bernstein, "Robust Stability and Dynamic Outputfeedback Stabilization: Deterministic and Stochastic Perspectives," IEEE Trans. Autom. Contr., pp 1076-1084, 1987.

[24] O. I. Kosmidou and P. Bertrand, "Robust-Controller Design for Systems with Large Parameter Variations," Int. J. Contr., Vol. 45, pp. 927-938, 1987.

[25] W. M. Wonham, Linear Multivariable Control: A Geometric Approach, New York: Springer-Verlag, 1979.

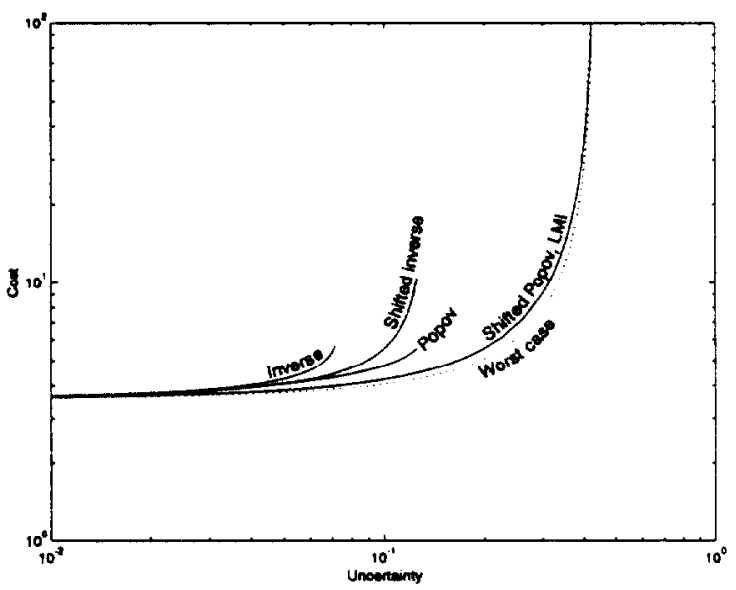

Figure 3: Cost bounds for Example 3.

\section{Appendix}

Proof of Theorem 1. Note that for all $\Delta A \in \mathcal{U},(5)$ is equivalent to

$$
\begin{aligned}
0= & (A+\Delta A)^{\mathrm{T}} P(A+\Delta A)-P \\
& +\Omega(P)-\left(\Delta A^{\mathrm{T}} P A+A^{\mathrm{T}} P \Delta A+\Delta A^{\mathrm{T}} P \Delta A\right)+R
\end{aligned}
$$

Adding and subtracting $(A+\Delta A)^{\mathrm{T}} P_{0}(\Delta A)(A+\Delta A)-P_{0}(\Delta A)$ to (42) yields

$$
\begin{aligned}
0= & (A+\Delta A)^{\mathrm{T}}\left(P+P_{0}(\Delta A)\right)(A+\Delta A)-\left(P+P_{0}(\Delta A)\right) \\
& +\Omega(P)-\left[(A+\Delta A)^{\mathrm{T}} P_{0}(\Delta A)(A+\Delta A)-P_{0}(\Delta A)\right] \\
& -\left(\Delta A^{\mathrm{T}} P A+A^{\mathrm{T}} P \Delta A+\Delta A^{\mathrm{T}} P \Delta A\right)+R .
\end{aligned}
$$

Hence, by assumption, (43) has a solution $P \in \mathcal{N}$ for all $\Delta A \in$ $\mathrm{R}^{n \times n}$. If $\triangle A$ is restricted to the set $U$, then, by (7), $\Omega(P)-$ $\left[(A+\Delta A)^{\mathrm{T}} P_{0}(\Delta A)(A+\Delta A)-P_{0}(\Delta A)\right]-\left(\Delta A^{\mathrm{T}} P A+A^{\mathrm{T}} P \Delta A+\right.$ $\left.\triangle A^{\mathrm{T}} P \triangle A\right)$ is nonnegative definite. Thus if $(A+\triangle A, E)$ is detectable for all $\Delta A \in \mathcal{U}$, then Theorem 3.6 of [25] implies $(A+$ $\left.\Delta A,\left[R+\bar{\Omega}(P, \Delta A)-\left(\Delta A^{\mathrm{T}} P A+A^{\mathrm{T}} P \Delta A+\Delta A^{\mathrm{T}} P \Delta A\right)\right]^{1 / 2}\right)$ is detectable for all $\Delta A \in \mathcal{U}$, where 
$\bar{\Omega}(P, \Delta A) \triangleq \Omega(P)-\left[(A+\Delta A)^{\mathbf{T}} P_{0}(\Delta A)(A+\Delta A)-P_{0}(\Delta A)\right]$.

It now follows from (43) and Lemma $12.2^{\prime}$ of [25] that $A+\Delta A$ is asymptotically stable for all $\Delta A \in \mathcal{U}$. Conversely, if $A+\Delta A$ is asymptotically stable for all $\triangle A \in \mathcal{U}$, then it follows immediately that $(A+\triangle A, E)$ is detectable for all $\triangle A \in \mathcal{U}$. Now, subtracting (4) from (43) yields

$$
\begin{aligned}
0= & (A+\Delta A)^{\mathrm{T}}\left(P+P_{0}(\Delta A)-P_{\Delta A}\right)(A+\Delta A) \\
& -\left(P+P_{0}(\Delta A)-P_{\Delta A}\right)+\Omega(P) \\
& -\left[(A+\Delta A)^{\mathrm{T}} P_{0}(\Delta A)(A+\Delta A)-P_{0}(\Delta A)\right] \\
& -\left(\Delta A^{\mathrm{T}} P A+A^{\mathrm{T}} P \Delta A+\Delta A^{\mathrm{T}} P \Delta A\right), \quad \Delta A \in \mathcal{U}
\end{aligned}
$$

or, equivalently, since $A+\Delta A$ is asymptotically stable for all $\Delta A \in$ $\mathcal{U}$

$$
\begin{aligned}
0 \leq & \sum_{k=0}^{\infty}(A+\Delta A)^{k \mathrm{~T}}[\bar{\Omega}(P, \Delta A) \\
& \left.-\left(\Delta A^{\mathrm{T}} P A+A^{\mathrm{T}} P \Delta A+\Delta A^{\mathrm{T}} P \Delta A\right)\right](A+\Delta A)^{k} \\
= & P+P_{0}(\Delta A)-P_{\Delta A}, \quad \Delta A \in \mathcal{U}
\end{aligned}
$$

which implies (8). The performance bounds (9) and (11) are now an immediate consequence of (3), (8), and (10).

Proof of Proposition 1. Let $\Delta A \in \mathcal{U}$, and let

$$
P=\hat{P}-\left(\bar{P}_{0}-\overline{\hat{P}}_{0}\right) \text {. }
$$

Then since $P \in \mathcal{N}$, it follows that $\hat{P} \in \hat{\mathcal{N}}$. Substituting (45) into (7), yields

$$
\begin{aligned}
& 0 \leq-\Delta A^{\mathrm{T}} P A-A^{\mathrm{T}} P \Delta A-\Delta A^{\mathrm{T}} P \Delta A+\Omega(P) \\
& -\left[(A+\Delta A)^{\mathrm{T}} P_{0}(\Delta A)(A+\Delta A)-P_{0}(\Delta A)\right] \\
& =-\Delta A^{\mathrm{T}}\left(\hat{P}-\bar{P}_{0}+\overline{\hat{P}}_{0}\right) A-A^{\mathrm{T}}\left(\hat{P}-\bar{P}_{0}+\overline{\hat{P}}_{0}\right) \Delta A \\
& -\Delta A^{\mathrm{T}}\left(\hat{P}-\bar{P}_{0}+\overline{\hat{P}}_{0}\right) \Delta A+\Omega\left(\hat{P}-\bar{P}_{0}+\overline{\hat{P}}_{0}\right) \\
& -\left[(A+\Delta A)^{\mathrm{T}} P_{0}(\Delta A)(A+\Delta A)-P_{0}(\Delta A)\right] \\
& =-\left(\Delta A^{\mathrm{T}} \hat{P} A+A^{\mathrm{T}} \hat{P} \Delta A+\Delta A^{\mathrm{T}} \hat{P} \Delta A\right)+\Omega\left(\hat{P}-\bar{P}_{0}+\hat{\hat{P}}_{0}\right) \\
& -\left[(A+\Delta A)^{\mathrm{T}} P_{0}(\Delta A)(A+\Delta A)-P_{0}(\Delta A)\right] \\
& +\Delta A^{\mathrm{T}}\left(\bar{P}_{0}-\overline{\hat{P}}_{0}\right) A+A^{\mathrm{T}}\left(\bar{P}_{0}-\overline{\hat{P}}_{0}\right) \Delta A+\Delta A^{\mathrm{T}}\left(\bar{P}_{0}-\overline{\hat{P}}_{0}\right) \Delta A \\
& =-\left(\Delta A^{\mathrm{T}} \hat{P} A+A^{\mathrm{T}} \hat{P} \Delta A+\Delta A^{\mathrm{T}} \hat{P} \Delta A\right)+\hat{\Omega}(\hat{P}) \\
& -\left[(A+\Delta A)^{\mathrm{T}} P_{0}(\Delta A)(A+\Delta A)-P_{0}(\Delta A)\right] \\
& +(A+\Delta A)^{\mathrm{T}}\left(\bar{P}_{0}-\overline{\hat{P}}_{0}\right)(A+\Delta A)-\left(\bar{P}_{0}-\overline{\hat{P}}_{0}\right) \\
& =-\left(\Delta A^{\mathrm{T}} \hat{P} A+A^{\mathrm{T}} \hat{P} \Delta A+\Delta A^{\mathrm{T}} \hat{P} \Delta A\right)+\hat{\Omega}(\hat{P}) \\
& -\left[(A+\Delta A)^{\mathrm{T}}\left(P_{0}(\Delta A)-\bar{P}_{0}+\overline{\hat{P}}_{0}\right)(A+\Delta A)\right. \\
& \left.-\left(P_{0}(\Delta A)-\bar{P}_{0}+\overrightarrow{\hat{P}}_{0}\right)\right] \\
& =-\left(\Delta A^{\mathrm{T}} \hat{P} A+A^{\mathrm{T}} \hat{P} \Delta A+\Delta A^{\mathrm{T}} \hat{P} \Delta A\right)+\hat{\Omega}(\hat{P}) \\
& -\left[(A+\Delta A)^{\mathrm{T}} \hat{P}_{0}(\Delta A)(A+\Delta A)-\hat{P}_{0}(\Delta A)\right]
\end{aligned}
$$

which completes the proof.

Proof of Proposition 4. Let $X=\left(T-B_{0}^{\mathrm{T}} P B_{0}\right)$. Then

$$
\begin{aligned}
0 \leq & {\left[X^{-1}\left(B_{0}^{\mathrm{T}} P A-H\right)-X F C_{0}\right]^{\mathrm{T}}\left[X^{-1}\left(B_{0}^{\mathrm{T}} P A-H\right)-X F C_{0}\right] } \\
& +Z-\left(H^{\mathrm{T}} F C_{0}+C_{0}^{\mathrm{T}} F^{\mathrm{T}} H+C_{0}^{\mathrm{T}} F^{\mathrm{T}} T F C_{0}\right) \\
= & \left(A^{\mathrm{T}} P B_{0}-H^{\mathrm{T}}\right) X^{-2}\left(B_{0}^{\mathrm{T}} P A-H\right)+C_{0}^{\mathrm{T}} F^{\mathrm{T}} X^{2} F C_{0} \\
& -C_{0}^{\mathrm{T}} F^{\mathrm{T}}\left(B_{0}^{\mathrm{T}} P A-H\right)+Z-\left(A^{\mathrm{T}} P B_{0}-H^{\mathrm{T}}\right) F C_{0} \\
& -\left(H^{\mathrm{T}} F C_{0}+C_{0}^{\mathrm{T}} F^{\mathrm{T}} H+C_{0}^{\mathrm{T}} F^{\mathrm{T}} T F C_{0}\right) \\
= & \left(A^{\mathrm{T}} P B_{0}-H^{\mathrm{T}}\right)\left(T-B_{0}^{\mathrm{T}} P B_{0}\right)^{-1}\left(B_{0}^{\mathrm{T}} P A-H\right) \\
& +C_{0}^{\mathrm{T}} F^{\mathrm{T}}\left(T-B_{0}^{\mathrm{T}} P B_{0}\right) F C_{0}-C_{0}^{\mathrm{T}} F^{\mathrm{T}} B_{0}^{\mathrm{T}} P A+Z \\
& -A^{\mathrm{T}} P B_{0} F C_{0}-C_{0}^{\mathrm{T}} F^{\mathrm{T}} T F C_{0}
\end{aligned}
$$

$$
\begin{aligned}
= & \left(A^{\mathrm{T}} P B_{0}-H^{\mathrm{T}}\right)\left(T-B_{0}^{\mathrm{T}} P B_{0}\right)^{-1}\left(B_{0}^{\mathrm{T}} P A-H\right)+Z \\
& -C_{0}^{\mathrm{T}} F^{\mathrm{T}} B_{0}^{\mathrm{T}} P A-A^{\mathrm{T}} P B_{0} F C_{0}-C_{0}^{\mathrm{T}} F^{\mathrm{T}} B_{0}^{\mathrm{T}} P B_{0} F C_{0} \\
= & \left(A^{\mathrm{T}} P B_{0}-H^{\mathrm{T}}\right)\left(T-B_{0}^{\mathrm{T}} P B_{0}\right)^{-1}\left(B_{0}^{\mathrm{T}} P A-H\right)+Z \\
& -\Delta A^{\mathrm{T}} P A-A^{\mathrm{T}} P \Delta A-\Delta A^{\mathrm{T}} P \Delta A \\
= & \Omega(P)-\Delta A^{\mathrm{T}} P A-A^{\mathrm{T}} P \Delta A-\Delta A^{\mathrm{T}} P \Delta A .
\end{aligned}
$$

which completes the proof.

Proof of Proposition 7. Define $X=(1-\alpha)(P-N) \geq 0$, and $A_{0}=\sum_{i=1}^{r} A_{i}$. Then

$$
\begin{aligned}
0 \leq & \alpha^{-1}\left(\sum_{i=1}^{r} \delta_{i} A_{i}^{\mathrm{T}}-A^{\mathrm{T}}(P-N) \alpha X^{-1}\right) \\
& \times X\left(\sum_{i=1}^{r} \delta_{i} A_{i}-\alpha X^{-1}(P-N) A\right) \\
= & \alpha A^{\mathrm{T}}(P-N) X^{-1}(P-N) A+\alpha^{-1}\left(\sum_{i=1}^{r} \delta_{i} A_{i}^{\mathrm{T}}\right) X\left(\sum_{i=1}^{r} \delta_{i} A_{i}\right) \\
& -\Delta A^{\mathrm{T}}(P-N) A-A^{\mathrm{T}}(P-N) \Delta A \\
\leq & \frac{\alpha}{1-\alpha} A^{\mathrm{T}}(P-N) A+\gamma^{2} \alpha^{-1} A_{0}^{\mathrm{T}}(P-N) A_{0} \\
& +\gamma^{2} A_{0}^{\mathrm{T}} N A_{0}+\gamma \sum_{i=1}^{r}\left|A_{i}^{\mathrm{T}} N A+A^{\mathrm{T}} N A_{i}\right| \\
& -\Delta A^{\mathrm{T}} P \Delta A-\Delta A^{\mathrm{T}} P A-A^{\mathrm{T}} P \Delta A \\
= & \Omega(P)-\Delta A^{\mathrm{T}} P A-A^{\mathrm{T}} P \Delta A-\Delta A^{\mathrm{T}} P \Delta A .
\end{aligned}
$$

which completes the proof.

\section{Proof of Proposition 11}

$0 \leq\left(\left[H C_{0}+\left(N C_{0}+H^{-1} B_{0}^{\mathrm{T}} C_{0}^{\mathrm{T}} H \mu C_{0}\right)\left(A_{\text {Pop }}-I\right)\right.\right.$

$$
\begin{aligned}
& \left.\left.+H^{-1} B_{0}^{\mathrm{T}} P A_{\mathrm{Pop}}-X B_{0}^{\mathrm{T}}\right]-R_{0} H\left(F-M_{L}\right) C_{0}\right)^{\mathrm{T}} R_{0}^{-1} \\
& \times\left(\left[H C_{0}+\left(N C_{0}+H^{-1} B_{0}^{\mathrm{T}} C_{0}^{\mathrm{T}} H \mu C_{0}\right)\left(A_{\mathrm{Pop}}-I\right)\right.\right. \\
& \left.\left.+H^{-1} B_{0}^{\mathbf{T}} P A_{\text {Pop }}-X B_{0}^{\mathrm{T}}\right]-R_{0} H\left(F-M_{L}\right) C_{0}\right) \\
& +\left[\left(A_{\text {Pop }}-I\right)+\Delta A-B_{0} M_{L} C_{0}\right]^{\mathrm{T}}\left(\vec{P}_{0}-P_{0}(F)\right) \\
& \times\left[\left(A_{\text {Pop }}-I\right)+\Delta A-B_{0} M_{L} C_{0}\right] \\
& +2 C_{0}^{\mathrm{T}} H\left(\left(F-M_{L}\right)-\left(F-M_{L}\right) M^{-1}\left(F-M_{L}\right)\right) H C_{0} \\
& -\left[B_{0} X^{\mathrm{T}}\left(F-M_{L}\right) H C_{0}+C_{0}^{\mathrm{T}} H\left(F-M_{L}\right) X B_{0}^{\mathrm{T}}\right] \\
& -\left(A_{\text {Pop }}-I\right)^{\mathrm{T}} C_{0}^{\mathrm{T}} H \mu C_{0}\left(A_{\text {Pop }}-I\right)+Z \\
& =\left[H C_{0}+\left(N C_{0}+H^{-1} B_{0}^{\mathrm{T}} C_{0}^{\mathrm{T}} H \mu C_{0}\right)\left(A_{\text {Pop }}-I\right)\right. \\
& \left.+H^{-1} B_{0}^{\mathrm{T}} P A_{\mathrm{Pop}}-X B_{0}^{\mathrm{T}}\right]^{\mathrm{T}} R_{0}^{-1}\left[H C_{0}-X B_{0}^{\mathrm{T}}\right. \\
& \left.+\left(N C_{0}+H^{-1} B_{0}^{\mathrm{T}} C_{0}^{\mathrm{T}} H \mu C_{0}\right)\left(A_{\text {Pop }}-I\right)+H^{-1} B_{0}^{\mathrm{T}} P A_{\text {Pop }}\right] \\
& -P_{0}(F)\left(\Delta A-B_{0} M_{L} C_{0}\right)-\left(\Delta A^{\mathrm{T}}-C_{0}^{\mathrm{T}} M_{L} B_{0}^{\mathrm{T}}\right) P_{0}(F) \\
& -\left(\Delta A^{\mathrm{T}}-C_{0}^{\mathrm{T}} M_{L} B_{0}^{\mathrm{T}}\right) P\left(\Delta A-B_{0} M_{L} C_{0}\right) \\
& -\left[\left(A_{\mathrm{Pop}}^{\mathrm{T}}-I\right) P_{0}(F)+A_{\mathrm{Pop}}^{\mathrm{T}} P\left(\Delta A-B_{0} M_{L} C_{0}\right)\right] \\
& -\left[P_{0}(F)\left(A_{\mathrm{Pop}}-I\right)+\left(\Delta A^{\mathrm{T}}-C_{0}^{\mathrm{T}} M_{L} B_{0}^{\mathrm{T}}\right) P A_{\mathrm{Pop}}\right] \\
& -[A-I+\Delta A]^{\mathrm{T}} P_{0}(F)[A-I+\Delta A]+Z \\
& =\left[H C_{0}-X B_{0}^{\mathrm{T}}+\left(N C_{0}+H^{-1} B_{0}^{\mathrm{T}} C_{0}^{\mathrm{T}} H \mu C_{0}\right)\left(A_{\text {Pop }}-I\right)\right. \\
& \left.+H^{-1} B_{0}^{\mathrm{T}} P A_{\mathrm{Pop}}\right]^{\mathrm{T}} R_{0}^{-1}\left[H C_{0}-X B_{0}^{\mathrm{T}}\right. \\
& \left.+\left(N C_{0}+H^{-1} B_{0}^{\mathrm{T}} C_{0}^{\mathrm{T}} H \mu C_{0}\right)\left(A_{\mathrm{Pop}}-I\right)+H^{-1} B_{0}^{\mathrm{T}} P A_{\mathrm{Pop}}\right] \\
& +A^{\mathrm{T}} P B_{0} M_{L} C_{0}+C_{0}^{\mathrm{T}} M_{L} B_{0}^{\mathrm{T}} P A+C_{0}^{\mathrm{T}} M_{L} B_{0}^{\mathrm{T}} P B_{0} M_{L} C_{0} \\
& +Z-\left(\triangle A^{\mathrm{T}} P \Delta A+A^{\mathrm{T}} P \Delta A+\Delta A^{\mathrm{T}} P A\right) \\
& -(A+\Delta A)^{\mathrm{T}} P_{0}(F)(A+\Delta A)+P_{0}(F) \\
& =\Omega_{0}(P)-\left(\Delta A^{\mathrm{T}} P A+A^{\mathrm{T}} P \Delta A+\Delta A^{\mathrm{T}} P \Delta A\right) \\
& -\left[(A+\Delta A)^{\mathrm{T}} P_{0}(F)(A+\Delta A)-P_{0}(F)\right]
\end{aligned}
$$

which completes the proof. 